\title{
ON THE BETTI NUMBERS OF IRREDUCIBLE COMPACT HYPERKÄHLER MANIFOLDS OF COMPLEX DIMENSION FOUR
}

\author{
Daniel Guan
}

\section{Introduction}

The study of higher dimensional hyperkähler manifolds has attracted much attention: we have [Wk], [Bg1,2,3,4], [Fj1,2], [Bv1], [Vb1,2], [S11,2], [HS], [Huy], $[\mathrm{Gu} 3,4,5]$ etc. It is evident that there are only a few known examples of these manifolds and the obvious question is: can we classify them as in the case of complex dimension 4 ?

The Riemann-Roch formula plays an important role in the surface case, which yields K-3 surfaces as the only irreducible examples. However, for the higher dimensional case, the Riemann-Roch formula is not enough to give a picture of both the Hodge diamond and the existence of holomorphic sections of line bundles. In [Gu5], we combined the results of the Riemann-Roch formula in [S11,2] (see also $[\mathrm{LW}]$ ) and the representations generated by the Kähler classes (see [Vb2], [LL], [Bg4]) to give a picture of the Hodge diamonds of irreducible compact hyperkähler manifolds of complex dimension 4 . Theorem 1 (reproduced here) gives an upper bound $b_{2} \leq 23$ for the second Betti number and was obtained independently by Beauville [Bv2] (unpublished). He kindly let me publish alone. The bound is obtained by applying Verbitsky's work. In [HS] there is also an upper bound for the Euler characteristic but there seems as yet no lower bound, nor any bound for the Betti numbers.

However, the method in [HS] actually gives us a way to calculate what we call generalized Chern numbers (which are only defined on hyperkähler manifolds) by Rozansky-Witten invariants, some of which in turn can be calculated as Chern numbers. Combining this approach with the method in [Bg4] we obtain an inequality in the opposite direction to the one in [HS] and apply it to our situation. Surprisingly, once we already have the bound on $b_{2}$ this gives a more natural and much stronger inequality than the one we manipulated from the Riemann-Roch formula in [Gu5].

Therefore, we obtain our:

Main Theorem. If $M$ is an irreducible compact hyperkähler manifold of complex dimension 4 , then $3 \leq b_{2} \leq 23$. Moreover,

Received June 7, 2000.

Supported by NSF Postdoctoral Fellowship DMS-9627434. 
1. if $b_{2}=23$, then $b_{3}=0$. The Hodge diamond of $M$ is the same as that of the Hilbert scheme of pairs of points on a K3 surface.

2. if $b_{2} \neq 23$, then $b_{2} \leq 8$, and when $b_{2}=8, b_{3}=0$.

3. in the case of $b_{2}=3,4,5,6$ then $b_{3}=4 l$ with $l \leq 17$ if $b_{2}=3, l \leq 15$ if $b_{2}=4, l \leq 9$ if $b_{2}=5$ and $l \leq 4$ if $b_{2}=6$.

4. in the case of $b_{2}=7, b_{3}=0$ or 8 .

5. the second Chern class $c_{2}$ lies in the algebra $H^{(4)}$ generated by $H^{2}(M)$ if and only if $\left(b_{2}, b_{3}\right)=(5,36),(7,8),(8,0),(23,0)$.

We may remark that $(7,8)$ and $(23,0)$ are the Betti numbers for the two known examples in this dimension. We doubt the possibility of existence of the case $b_{2}=3$ although much more work should be done in this direction.

Although we apply some technical tools in this paper, we hope that it contains ideas which will be useful for complex dimension $\geq 6$ in the future.

To make the material easier for the reader, we first give an easy proof of the bounds on $b_{2}$ in Section 2. Then, we give a proof of the new inequality which gives most information about the restrictions on both $b_{2}$ and $b_{3}$, except for the case of $b_{2}=7$ (which we dealt with in [Gu5]), in Section 3. Section 3 is the core of this paper. We have given some examples of the possible Hodge algebras as Jordan-Lefschetz modules (see [LL]) in [Gu5].

\section{Bound on $b_{2}$}

Given a K3 surface $K$, Fujiki in [Fj1] constructed an irreducible compact complex 4 dimensional hyperkähler manifold $K_{[2]}$ by blowing up the diagonal of $K^{(2)}=K \times K / S_{2}$ with $S_{2}$ the symmetric group of two elements. This is in Beauville's language $[\mathrm{Bv} 1]$ the Hilbert scheme of pairs of points. The second Betti number of $K_{[2]}$ is 23 and the third Betti number is 0 .

Theorem 1. If $M$ is an irreducible compact hyperkähler manifold of complex dimension 4 , then $b_{2} \leq 23$. The maximum of $b_{2}$ is achieved by $K_{[2]}$. And if $b_{2}=23$ the Hodge diamond is the same as that of $K_{[2]}$.

Proof. In [Sl2] (see also [Hz p.6], [Sl1 p.117] for complex dimension 4 and [LW] for a formula of Hodge numbers) Salamon obtained a Riemann-Roch formula for irreducible compact hyperkähler manifolds of complex dimension $2 \mathrm{~m}$ :

$$
2 \sum_{j=1}^{2 m}(-1)^{j}\left(3 j^{2}-m\right) b_{2 m-j}=m b_{2 m} .
$$

In the case $m=2$ and $b_{1}=0$ we have

$$
b_{3}+b_{4}=46+10 b_{2} \text {. }
$$

By the result in [Vb2] (see also [LL], [Bg4]) the second symmetric product of $H^{2}(M)$ maps injectively by cup product into the cohomology group $H^{4}(M)$ so we have

$$
b_{4} \geq \frac{b_{2}\left(b_{2}+1\right)}{2} .
$$


Therefore,

$$
b_{2}^{2}+b_{2} \leq 92+20 b_{2}
$$

i.e., $b_{2}^{2}-19 b_{2}-23 \times 4 \leq 0$. We obtain $\left(b_{2}+4\right)\left(b_{2}-23\right) \leq 0$, i.e., $b_{2} \leq 23$.

If $b_{2}=23$, then $b_{4} \geq 23 \times 12=276$. Therefore, $b_{3}+276 \leq 46+230$, i.e., $b_{3}=0$.

\section{The Generalized Chern Numbers and Riemann-Roch Formula}

Let $M$ be a compact hyperkähler manifold, $C$ be a polynomial in the (necessarily even) Chern classes of degree $4 r$. A consequence of the results of Fujiki in $[\mathrm{Fj} 2]$ is:

Lemma 1. The number $N(C)=\int_{M} C u^{2 n-2 r} /\left(\int_{M} u^{2 n}\right)^{\frac{n-r}{n}}$ is independent of $u \in H^{2}(M)$ with $v(u)=\int_{M} u^{2 n} \neq 0$.

Proof. By Theorem 4.7 and Lemma 4.11 in [Fj2] (see also [Gu3 Proposition 1], [Gu4 Theorem 4] and [LL Theorem 4.7]), we have

$$
c(u)=\int_{M} C u^{2 n-2 r}=a Q^{n-r}(u), v(u)=b Q^{n}(u)
$$

with $Q(u)$ the rational quadratic form on $H^{2}(M)$ defined in [Bg3,4], [Bv1] (see also [Gu4 Theorem 4] and [Huy]) and $b>0$. Therefore, $N(C)=c(u) v^{-\frac{n-r}{n}}(u)=$ $a b^{-\frac{n-r}{n}}$ is a constant.

We call $N(C)$ a generalized Chern number of degree $r$ (see [BN] for a recent discussion of these). When $r=n$, we have the ordinary Chern numbers. In [HS] Hitchin and Sawon calculated the generalized Chern number with $C=c_{2}$. By the stability of the tangent bundle it is not difficult to see that $N\left(c_{2}\right)>0$; also a standard formula in Riemannian geometry leads to an equality with the $L^{2}$ norm of the curvature. It is not difficult to see that all of the generalized Chern numbers can be interpreted as Rozansky-Witten invariants ([BN]). These can sometimes be calculated as Chern numbers. This is certainly true for complex dimension 4 and 6 because for $n=4$ there are only three interesting trivalent graphs with one nontrivial IHX relation and so there are only two independent Rozansky-Witten invariants, the same as the number of independent degree 8 Chern polynomials. Similarly for $n=6$ there are five interesting connected degree three trivalent graphs with four nontrivial IHX relations. It is also very interesting that each of these relations represents one graph as twice of another graph. Therefore, these relations produce identities with powers of 2 .

Here we state Hitchin and Sawon's Theorem in our language.

Lemma 2. $\frac{((2 n) !)^{n-1} N\left(c_{2}\right)^{n}}{(24 n(2 n-2) !)^{n}}=\sqrt{\hat{A}}[M]$.

This Riemann-Roch type formula from [HS] enable us to manipulate our generalized Chern numbers. An inequality for $N\left(c_{2}\right)$ in the opposite direction to $N\left(c_{2}\right)>0$ is given by the following (where we denote by $H^{(*)}=\oplus_{m} H^{(2 m)}$ the subalgebra of $H^{*}(M)$ generated by $\left.H^{2}(M)\right)$. 
Lemma 3. If $M$ is an irreducible compact hyperkähler manifold of complex dimension 4 , then

$$
3 b_{2} N\left(c_{2}\right)^{2} \leq\left(b_{2}+2\right) c_{2}^{2}[M]
$$

with equality if and only if $c_{2}$ lies in $H^{(4)}$.

Proof. Here we follow the argument in [Bg4]. Let $Q=\left(Q_{i j}\right)$ be the BogomolovBeauville quadratic form on $H^{2}(M)$ and $q$ its dual $\left(q_{i j}\right)=\left(Q_{i j}\right)^{-1}$. So $q \in$ $\operatorname{Sym}^{2} H^{2}(M)$ and by exterior multiplication defines a class we still call $q$ in $H^{4}(M)$. Since $c_{2}$ is a Pontryagin class and thus independent of any complex structure it follows from $[\mathrm{Bg} 4]$ that its projection (using the intersection form) of $c_{2}$ in $H^{(4)}$ is a multiple $p=\lambda q$ of $q$. We write $c_{2}=p+p^{\perp}$. The orthogonal complement to $H^{(4)}$ in $H^{4}(M)$ consists of primitive forms. Since $c_{2}$ and $q$ are of type $(2,2)$, by the Hodge-Riemann bilinear relations (see [GH p.123]) the intersection form is positive on $p^{\perp}$ and so

$$
\int_{M} c_{2}^{2}=\int_{M} p^{2}+\left(p^{\perp}\right)^{2} \geq \int_{M} p^{2} .
$$

But $\int_{M} p^{2}=\lambda^{2} \int_{M} q^{2}=\lambda \int_{M} c_{2} q$ and so $\int_{M} c_{2}^{2} \geq\left(\int_{M} c_{2} q\right)^{2} / \int_{M} q^{2}$. Take an orthonormal basis $\left\{e_{i}\right\}$ of $H^{2}(M)$ for the quadratic form $Q$ over the complex numbers, then $q=\sum_{1}^{n} e_{i}^{2}$ where $n=b_{2}$. Since $\int_{M} u^{4}=b Q(u)^{2}$ and $Q\left(e_{i}\right)=1$ we have for $i \neq j, \int_{M}\left(e_{i}+e_{j}\right)^{4}=4 b=\int_{M}\left(e_{i}-e_{j}\right)^{4}$ and since $\int_{M} e_{i}^{4}=b$ it follows that $\int_{M} e_{i}^{2} e_{j}^{2}=b / 3$. Thus

$$
\int_{M} q^{2}=\int_{M}\left(\sum e_{i}^{2}\right)^{2}=n(n-1) \frac{b}{3}+n b=\frac{n(n+2)}{3} b
$$

and

$$
\int_{M} c_{2} q=\int_{M} c_{2} \sum e_{i}^{2}=n N\left(c_{2}\right) b^{1 / 2} .
$$

Therefore, the inequality can be written

$$
c_{2}^{2}[M] \geq 3 n N\left(c_{2}\right)^{2} /(n+2)=3 b_{2} N\left(c_{2}\right)^{2} /\left(b_{2}+2\right)
$$

Remark. For higher dimensions we can still apply $N\left(c_{2}^{2}\right)$ instead of $c_{2}^{2}[M]$ to obtain a generalization of this lemma and therefore obtain a generalization of the next theorem. This can be done since $N\left(c_{2}^{2}\right)$ can be calculated, as functions of Rozansky-Witten invariants, from the Chern numbers (we can see this above for $n=6)$. For a general $n$, it is done in [Gu6].

Theorem 2. If $M$ is an irreducible compact hyperkähler manifold of complex dimension 4 , then

$$
b_{3} \leq \frac{4\left(23-b_{2}\right)\left(8-b_{2}\right)}{b_{2}+1} .
$$

In particular since $b_{2} \leq 23$, if $b_{2}>7$, we have only the two cases: $\left(b_{2}, b_{3}\right)=$ $(8,0),(23,0)$. 
Proof. Applying Lemmas 2 and 3 we obtain:

$$
3 b_{2} \frac{(24 n(2 n-2) !)^{n}}{(2 n) !} \sqrt{\hat{A}}[M] \leq\left(b_{2}+2\right) c_{2}^{2}
$$

with $n=2$. Applying the Riemann-Roch formula as in [HS] we obtain:

$$
\begin{gathered}
\sqrt{\hat{A}}[M]=\frac{1}{2} \hat{A}_{2}[M]-\frac{1}{8} \hat{A}_{1}^{2}[M], \\
\hat{A}_{1}=\frac{1}{12} c_{2}, \\
\hat{A}_{2}[M]=\frac{1}{720}\left(3 c_{2}^{2}-c_{4}\right)[M]=3, \\
\mathcal{X}^{1}=h^{1,2}-2 h^{1,1}=12-\frac{c_{4}[M]}{6} .
\end{gathered}
$$

We have:

$$
\begin{gathered}
c_{4}[M]=3\left(24-b_{3}+4\left(b_{2}-2\right)\right)=3\left(4 b_{2}+16-b_{3}\right), \\
c_{2}^{2}[M]=720+\left(4 b_{2}-b_{3}+16\right)=736+4 b_{2}-b_{3} .
\end{gathered}
$$

Therefore,

$$
\begin{gathered}
3 b_{2} \frac{(24 \times 4)^{2}}{24} \sqrt{\hat{A}}[M]=2(24)^{2} b_{2}\left(\frac{3}{2}-\frac{c_{2}^{2}[M]}{144 \times 8}\right) \\
=b_{2}\left(3(24)^{2}-c_{2}^{2}[M]\right) \leq\left(b_{2}+2\right) c_{2}^{2}[M]
\end{gathered}
$$

Hence,

$$
3(24)^{2} b_{2} \leq 2\left(b_{2}+1\right)\left(736+4 b_{2}-b_{3}\right)
$$

i.e.,

$$
\begin{aligned}
& \left(b_{2}+1\right) b_{3} \leq 4\left(\left(b_{2}+184\right)\left(b_{2}+1\right)-216 b_{2}\right) \\
& \quad=4\left(b_{2}^{2}-31 b_{2}+23 \times 8\right)=4\left(23-b_{2}\right)\left(8-b_{2}\right)
\end{aligned}
$$

as desired

By the results in $[\mathrm{Wk}]$ (see also $[\mathrm{Fj} 2]$ ) we have $b_{3}=4 l$. Therefore, we have:

Corollary 1. $l \leq 17$ if $b_{2}=3$ and $l \leq 15$ if $b_{2}=4: l \leq 9$ if $b_{2}=5$ and $l \leq 4$ if $b_{2}=6$. When $b_{2}=7, l$ is either 0 or 2 . Moreover, $C_{2} \in H^{(4)}$ if and only if $\left(b_{2}, b_{3}\right)=(5,36),(7,8),(8,0),(23,0)$.

Proof. Applying the same argument as in the proof of Theorem 2, from

$$
b_{3}+b_{4}=46+10 b_{2}
$$

we obtain

$$
b_{3}+\frac{b_{2}\left(b_{2}+1\right)}{2} \leq 46+10 b_{2}
$$

Therefore, $2 b_{3} \leq 92+19 b_{2}-b_{2}^{2}$, i. e.,

$$
2 b_{3} \leq\left(23-b_{2}\right)\left(b_{2}+4\right) \text {. }
$$

If $b_{2}=3$, we have $8 l \leq 20 \times 7$, i.e., $l \leq \frac{35}{2}$. But $l$ is an integer, we obtain $l \leq 17$. 
If $b_{2}=4$, we apply Theorem 2 . Therefore,

$$
l \leq \frac{\left(23-b_{2}\right)\left(8-b_{2}\right)}{b_{2}+1},
$$

i.e., $l \leq \frac{19 \times 4}{5}<16$ and $l \leq 15$.

If $b_{2}=5$, we have $l \leq 9$.

If $b_{2}=6$, we have $l \leq \frac{17 \times 2}{7}<5$ and $l \leq 4$.

If $b_{2}=7$, we have $l \leq 2$. We have already got rid of the possibility of $l=1$ in [Gu5] with a method motivated from [Gu1,2]. This is also an important statement, it means that if $b_{2}=7$, then we have the Hodge diamond of the Kummer variety or $b_{3}=0$.

The equality holds only in those four cases in the Corollary as desired.

\section{Acknowledgement}

We thank the Department of Mathematics of Princeton University and the University of California at Riverside for their support. I also thank NSF for the fellowship which made this work possible, although the breakthrough came only at the last moment.

We thank Professors F. Bogomolov and S. Kobayashi, Y. Siu for their constant support. Professor Kobayashi also told me about [Kr] and [Wk].

We thank Professors M. Gromov and D. Joyce for showing me [Sl] while I was explaining [Gu3], and Professor T. Springer for showing me [KMRT] while he was reading [Gu1], which eventually led me to apply the spinor theory in [Gu5]. We also thank Professor Beauville for showing me [LW] and the referees for mentioning $[\mathrm{HS}],[\mathrm{BN}]$ and Beauville's results.

\section{References}

[Bg1] F. Bogomolov. Kähler manifolds with trivial canonical class, Izv. Akad. Nauk SSSR Ser. Mat. 38 (1974), 11-21.

[Bg2] F. Bogomolov. The decomposition of Kähler manifolds with a trivial canonical class, Mat. Sb. (N.S.) 93 (135) (1974), 573-575, 630.

[Bg3] F. Bogomolov. Hamiltonian Kählerian manifolds, Dokl. Akad. Nauk SSSR 243 (1978), no. 5, 1101-1104.

[Bg4] F. Bogomolov. On the cohomology ring of a simple hyperkähler manifold (on the results of Verbitsky), Geom. Funct. Anal. 6 (1996), 612-618.

[Bv1] A. Beauville. Variétés Kähleriennes dont la premir̀e classe de Chern est nulle, J. Differential Geometry 18 (1983), no. 4, 755-782.

[Bv2] A. Beauville. private communication, 2000.

[BN] M. Britze and M. A. Nieper. Hirzebruch-Riemann-Roch formulae on irreducible symplectic Kähler manifolds, arXiv: math.AG/0101062

[Fj1] A. Fujiki. On primitively symplectic compact Kähler $V$ manifolds of dimension four, Classification of algebraic and analytic manifolds (Katata 1982), 71-250. Progr. Math. 39, Birkhäuser, Boston (1983).

[Fj2] A. Fujiki. On the de Rham cohomology group of a compact Kähler symplectic manifold, Algebraic geometry, Sendai, 1985, 105-165, Adv. Stud. Pure Math., 10, NorthHolland, Amsterdam, 1987. 
[GH] P. Griffiths \& J. Harris, Principles of Algebraic Geometry, Pure and Applied Mathematics. Wiley-Interscience (John Wiley \& Sons), New York, 1978.

[Gu1] D. Guan, Toward a Classification of Compact Complex Homogeneous Spaces, preprint 1998.

[Gu2] Z. Guan, Toward a Classification of Almost Homogeneous Manifolds ILinearization of the Singular Extremal Rays, Internat. J. Math. 8 (1997), no. 8, 999-1014.

[Gu3] D. Guan, Examples of compact holomorphic symplectic manifolds which are not Kählerian II, Invent Math. 121 (1995), 135-145.

[Gu4] D. Guan, Examples of compact holomorphic symplectic manifolds which are not Kählerian III, Internat. J. Math. 6 (1995), 709-718.

[Gu5] D. Guan, On Riemann-Roch Formula and Bounds of the Betti Numbers of Irreducible Compact Hyperkähler Manifold-n=4, preprint 1999.

[Gu6] D. Guan, An inequality for certain Rozansky-Witten invariants on compact irreducible hyperkähler manifolds, in preparation.

[HS] N. Hitchin and J. Sawon, Curvature and Characteristic Numbers of Hyperkähler Manifolds, Duke Mathematical J. 106 (2001), no. 3, 599-615.

[Huy] D. Huybrechts, Compact hyper-Kähler manifolds: basic results, Invent. Math. 135 (1999), no. 1, 63-113.

[Hz] F. Hirzebruch, Topological Methods in Algebraic Geometry, Grundlehren der mathematischen Wissenschaften 131, Springer Verlag, Heidelberg, 1978.

[KMRT] M. Knus, A. Merkurjev, M. Rost \& J. Tignol, The Book of Involutions, American Mathematical Society Colloquium Publications, 44, 1998.

[Kr] V. Kraines, Topology of quaternionic manifolds, Trans. Amer. Math. Soc. 122 (1966), 357-367.

[KL] A. Libgober \& J. Wood, Uniqueness of the complex structure on Kähler manifolds of certain homotopy types, J. Differential Geom. 32 (1990), no. 1, 139-154.

[LL] E. Looijenga \& V. Lunts, A Lie algebra attached to a projective variety, Invent. Math. 129 (1997), no. 2, 361-412.

[OG] K. O'Grady, Desingularized moduli spaces of sheaves on a K3, J. Reine Angew. Math. 512 (1999), 49-117.

[S11] S. Salamon, Riemannian Geometry and Holonomy Groups. Pitman Research Notes in Mathematics Series 201, Longman Scientific, Harlow, 1989.

[S12] S. Salamon, On the cohomology of Kähler and hyperkähler manifolds, Topology 35 (1996), no. 1, 137-155.

[Vb1] M. Verbitsky, Action of the Lie algebra of $S O(5)$ on the cohomology of a hyperkähler manifold, Funct. Anal. Appl. 24 (1991), 229-230.

[Vb2] M. Verbitsky, Cohomology of compact hyperkähler manifolds and its applications, Geom. Funct. Anal. 6 (1996), no. 4, 601-611.

[Wk] H. Wakakuwa, On Riemannian manifolds with homogeneous holonomy group $\operatorname{Sp}(n)$, Tôhoku Math. J. (2) 10 (1958), 274-303.

Department of Mathematics, The University of California at Riverside, RiverSIDE, CA 92521.

E-mail address: zguan@math.ucr.edu 